\title{
Rheology and structural arrest of casein suspensions
}

\author{
L. Dahbi ${ }^{\mathrm{a}, \mathrm{b}, *}$, M. Alexander ${ }^{\mathrm{a}}$, V. Trappe $^{\mathrm{a}}$, J.K.G. Dhont ${ }^{\mathrm{b}}$, P. Schurtenberger ${ }^{\mathrm{c}}$ \\ ${ }^{a}$ Department of Physics, University of Fribourg, CH-1700 Fribourg, Switzerland \\ ${ }^{\mathrm{b}}$ Forschungszentrum Jülich, IFF/Weiche Materie, D-52425 Jülich, Germany \\ ${ }^{\mathrm{c}}$ Adolphe Merkle Institute, University of Fribourg, $\mathrm{CH}-1700$ Fribourg, Switzerland
}

\begin{abstract}
The rheology of milk powder suspensions is investigated up to very high concentrations, where structural arrest occurs. The main component of the milk powder investigated is casein, so that the suspensions can be regarded as casein suspensions. Four concentration regimes are identified. For effective casein volume fractions less than 0.54 the concentration dependence of the zero-shear viscosity is similar to that of hard-sphere suspensions. However, due to the elastic deformation of the caseins, the viscosity does not diverge at the hard sphere glass transition. In the volume-fraction range of $0.55-0.61$ the viscosity exhibits a surprisingly weak dependence on concentration. The shape of the curve of the shear viscosity versus concentration deviates from hard sphere behavior in an unusual way, due to the observation of a region of almost constant viscosity. This concentration regime is followed by a regime where the viscosity steeply increases, eventually diverging at an effective volume fraction of 0.69 . Frequency dependent rheology and diffusing wave spectroscopy measurements indicate that the suspensions are jammed for volume fractions above 0.69. Finally we found the concentration dependence of the relative zero-shear viscosity of casein suspensions to be very similar with the one of the micro-gels at volume fractions below 0.50 and above 0.55 , which are know to shrink above a certain volume fraction, due to osmotic stress.
\end{abstract}

\section{Introduction}

Food science and technology have strongly benefited from recent developments in soft condensed matter. Important progress has in particular been made by drawing analogies between food colloids and colloidal model systems, their interactions and the resulting phase behavior [1]. These issues are of central relevance to food science and technology and have already considerably improved our understanding of food materials. An area that has received growing attention centers around fluid-solid transitions such as the glass, jamming or gelation transition that are frequently encountered in colloidal suspensions. Typical examples of arrested states of soft matter in food are yoghurt or cheese that can be seen as the food scientists analog of sol-gel processing in modern ceramics formation $[1,2]$.

Milk suspensions are indeed classical examples of food systems where analogies with colloidal model systems have frequently been invoked. Skim milk can for example be seen as a colloidal suspension of caseins, formed via self-assembly of four casein proteins and calcium phosphate, which are dispersed in an aqueous solu-

* Corresponding author. Address: Forschungszentrum Jülich, IFF/Weiche Materie, D-52425 Jülich, Germany. Fax: +49 4089984475.

E-mail address: Louisa.Dahbi@gmail.com (L. Dahbi). tion of salts, lactose and whey proteins [3,4]. The colloid analogy allows us to better understand and rationalize observations made in industrial processes such as the production of milk powder via spray drying frequently used in order to preserve milk for future use. The industrial spray-drying procedure involves full fat milk, whose water content is reduced by film evaporation until reaching a solid content of typically $50 \%$. Once this high solid content is achieved, the suspensions are subsequently homogenized by high pressure filtering. During this homogenization process the size of the fat globules and the casein micelles is reduced to about $400-600 \mathrm{~nm}$ and $300 \mathrm{~nm}$, respectively, in order to increase the stability of the suspension. By subsequent spray drying, the water content is further decreased by evaporation to about $65 \%$ solid content. In order to decrease the financial costs of this procedure, the solid content prior to spray drying should be as high as possible. However, above a solid content of $50 \%$ the viscosity of concentrated milk increases drastically, which renders homogenization through high pressure filtering problematic.

The increase of the viscosity of suspensions with increasing solid content is a well-known feature shared by all colloidal suspensions. In particular, de Kruif $[5,6]$ showed that suspensions of caseins closely follow the behavior of model hard-sphere suspensions. Both the concentration dependence of the viscosity and the self- and collective-diffusion coefficients of the casein 
suspensions are the same as for hard spheres up to volume fractions $\Phi \sim 0.25$. Alexander et al. [7] investigated different types of milk powder suspensions by diffusing wave spectroscopy and showed that the short-time collective-diffusion coefficient is in good agreement with that of hard spheres up to a volume fraction $\Phi \sim 0.45$. Each type of milk, however, displays its own onset of deviation from hard-sphere behavior above a certain volume fraction. This behavior is not unexpected as, unlike model hard spheres, caseins are loose self-assembled structures. Despite the numerous structural investigations (by small-angle X-ray and neutron scattering), the exact structure of the casein is not yet established and still debated [4,8-10]. This loose structure of the caseins apparently does not give rise to deviations from hardsphere behavior up to volume fractions of about 0.25 . At higher packing fractions, however, the deformability of casein is expected to play an important role both in the viscous behavior of the suspensions as well as the dynamics of the caseins.

We have thus conducted a systematic investigation of the rheology and dynamics of casein suspensions at very high packing fractions, where elastic deformation of caseins leads to strong deviations from hard-sphere behavior. The terminology "hard sphere" has two meanings here. It refers both to the hard-sphere type of interactions between the colloids as well as the essentially nondeformability of the core of single colloidal particles. The phenomena studied here for suspensions of caseins are similar to those in other suspensions of deformable colloids like micro-gel particles [11] or red blood cells [12]. Structural arrest of caseins will be seen to occur at much higher volume fractions as compared to hard spheres.

\section{Materials and experimental methods}

In this section we introduce the materials that we use and the way in which the casein suspensions are prepared. In addition, we describe the rheology and diffusing wave spectroscopy experiments performed to characterize the mechanical and dynamical properties of our systems.

\subsection{Material}

The milk powder used for the study described in this paper is Promilk 852 B from Cremo, Fribourg, Switzerland. The main characteristic of this powder is its high content of casein and its very small amount of lactose and fat : $81 \%$ protein, of which $92 \%$ is casein and $8 \%$ serum proteins (beta-lactoglobulin, alpha-lactalbumin, bovine serum albumin (BSA), immunoglobulin G (IgG) and phospholipoproteins), $4 \%$ lactose, $8.5 \%$ minerals, $1.5 \%$ fat and $5 \%$ water. As compared to other types of milk, this milk is essentially lactose free and fat free. To ensure reproducibility of our results, two different batches were studied, which we refer to as batches I and II. The polydispersity of both batches was found to be $\sigma=0.2$.

\subsection{Preparation of casein-micelle suspensions}

Milk suspensions were prepared in a range of casein-weight percent ([casein]) of $1.14-19.10 \%$ using the following procedure: de-ionized water is heated to $60{ }^{\circ} \mathrm{C}$ and the desired amount of powder is gradually added while stirring at moderate speed. The sample is then sealed and left to cool to $22{ }^{\circ} \mathrm{C}$ to fully equilibrate before the measurements.

In the low concentration range the volume fraction $\Phi$ of a casein suspension could in principle be obtained by multiplying the concentration in $\mathrm{g} / \mathrm{ml}$, by a factor $q$ that accounts for the voluminosity of the casein in the suspending medium
$\Phi=$ conc $\times q, \quad$ conc $=\frac{\text { weight of casein }}{\text { volume of the solution }}$

However, it has been shown that the values of $q$ significantly varies depending on the method used, such as microscopy, viscometry, sedimentation experiments or dynamic light scattering $[5,17]$. Moreover, due to the fact that casein is in our milks not the only component taking up space and that the relative composition varies from batch to batch, we chose to perform this study by focusing on the casein weight content. The concentration dependence of the viscosities of our milk powder suspensions is then compared to the volume fraction dependence of hard spheres [18-20] in the low to moderate concentration range, yielding an approximate value for the effective volume fraction and the factor $q$.

\subsection{Rheology measurements}

The shear rate dependent viscosity and the linear viscoelastic response functions were measured with a commercial stressstrain rheometer (MCR 300 Paar Physica). To ensure that the minimum torque of $0.5 \mu \mathrm{Nm}$ was maintained we used three different geometries. For the lowest casein concentrations a double-gap Couette cell was used, for the moderate concentrations a single gap Couette cell. At the highest concentration, where the use of a Couette cell becomes impracticable because the dispersion is not easily poured anymore, we used a cone-plate geometry with a cone-plate angle of $1^{\circ}$. Before each set of experiments, two viscosity standards (Newtonian oils furnished with the rheometer) in the relevant range of viscosity were measured to insure a precise calibration procedure, and we checked that the data obtained with a Couette cell and a cone-plate geometry superimpose. To ensure that wall slip is not significant we investigated a few concentrations with both geometries, Couette and cone-plate, where we found that the results obtained were essentially identical; this indicated that wall slip is indeed negligible in our experiments. Moreover, the frequency dependence of the measured storageand loss-modulus over the entire range of concentrations and frequencies indicates that no wall slip occurs during our measurements. When slippage occurs, the loss-modulus $G^{\prime \prime}$ should exhibit severe oscillations, which is not the case here. Prior to each measurement the samples were pre-sheared at $1000 \mathrm{~s}^{-1}$ for at least 2 min to erase any shear history. Visual inspection of our samples during unloading and the good reproducibility of our experiments revealed that our sample did not fracture despite the rather high shear rate applied. Viscosity measurements at a constant shear rate were performed, where the viscosity is monitored over extended periods of time to verify steady state conditions. Flow curves are obtained first by decreasing the shear rate from $1000 \mathrm{~s}^{-1}$ to lower shear rates and subsequently by increasing the shear rate again to $1000 \mathrm{~s}^{-1}$. In all cases the flow curves measured during a series of measurements on lowering the shear rate were, within experimental error (3\%), identical to the ones obtained on increasing the shear rate. This ensures that the measured viscosities are independent of the history of the samples. For the highest volume fraction, the lowest shear rate applied is $10^{-5} \mathrm{~s}^{-1}$, and several minutes were necessary to reach the steady state conditions. After such a series of measurements, the sample was submitted to a series of amplitude sweeps to determine the extent of the linear viscoelastic regime. We typically tested a strain range of $0.1 \%$ to $100 \%$ at three different angular frequencies, 1,10 and $100 \mathrm{rad} / \mathrm{s}$. For all our samples, frequency dependent measurements were done with strains between $0.5 \%$ and $2 \%$ within the linear response regime. Finally, to be able to account for the contributions of the background fluid, the viscosity of the milk suspensions without the casein micelles, the milk serum, is measured for each batch. To obtain the milk serum, the sample was passed directly through a Vivaflow 50 ultrafil- 
tration unit with $10 \mathrm{kD}$ molecular weight cutoff (Sartorius AG, Switzerland).

\subsection{Diffusing wave spectroscopy}

Natural milk as well as more concentrated casein suspensions are highly turbid media and cannot be investigated using conventional dynamic light scattering (DLS). To obtain insight in the dynamics of our suspensions, we thus used diffusing wave spectroscopy (DWS) [21,22]. This technique, which can be considered as an extension of DLS to the limit of strong multiple scattering, was successfully used in the past to investigate dairy products [23-27]. The principles of both DLS and DWS are based on the analysis of the fluctuations of scattered light intensities. In DLS, however, an unambiguous interpretation of data requires the absence of multiple scattering. By contrast, DWS probes the other extreme, where the scattered intensity originates essentially only from multiple scattering events that involve many colloidal particles. Since a photon traversing the sample is now scattered from many particles, very small displacements of the many particles along the scattering path give rise to an appreciable phase shift of the detected electric field; DWS thus typically probes the short-time dynamics of a system. We used a commercial DWS apparatus (Rheolab, LS Instruments [28]), in which the two-cell method is implemented to ensure a proper ensemble average of the intensity auto-correlation function [29-31]. The measurements were performed at a laser wavelength of $632.8 \mathrm{~nm}$. The sample cell was a rectangular Hellma cell with $L=5 \mathrm{~mm}$ path length, which is mounted in a temperature controlled sample holder at $22^{\circ} \mathrm{C}$. For all our samples $L$ is much larger than the transport mean-free path $l^{\star}$, which ensures that a photon effectively undergoes a random walk as it traverses the sample. The sample is loaded at room temperature after the equilibrium steps that were described in Section 2.2 and is subsequently left for $15 \mathrm{~min}$. in the Hellma cell at $22{ }^{\circ} \mathrm{C}$ prior to measurement. Scattered light is collected with a photo-multiplier in transmission and fed to a PC-interface autocorrelator, which evaluates the intensity auto-correlation function $g_{2}$. From the intensity auto-correlation function $g_{2}$, the electric field correlation function $g_{1}$ is obtained through the Siegert relation $g_{2}=1+g_{1}^{2}$.

\section{Rheology}

In this section we present first the concentration dependent zero-shear viscosities, which are found to diverge at relatively high effective volume fractions. Oscillatory rheology measurements are used to show that at this concentration the samples become solidlike, indicating that structural arrest occurs.

\subsection{Flow curves and zero-shear viscosities}

The viscosity is measured as a function of the shear rate over a large concentration range, up to concentrations were structural arrest occurs. The shear rate dependence of the viscosity normalized by the serum viscosity is given for various casein concentrations of batch II in Fig. 1. At low concentrations ([casein] $<8 \%$ ), the measured steady-shear viscosity is essentially shear-rate independent within the accessible shear-rate range. At higher concentrations the suspensions exhibits shear thinning. As for hard-sphere suspensions, the viscosity increases and the shear-thinning behavior becomes more pronounced with increasing casein concentration. The low-shear viscosity $\eta_{0}$ is extracted from a fit of the experimental viscosity data to the Cross-equation [32],

$\eta=\eta_{\infty}+\frac{\eta_{0}-\eta_{\infty}}{1+\left(\dot{\gamma} / \dot{\gamma}_{c}\right)^{m}}$

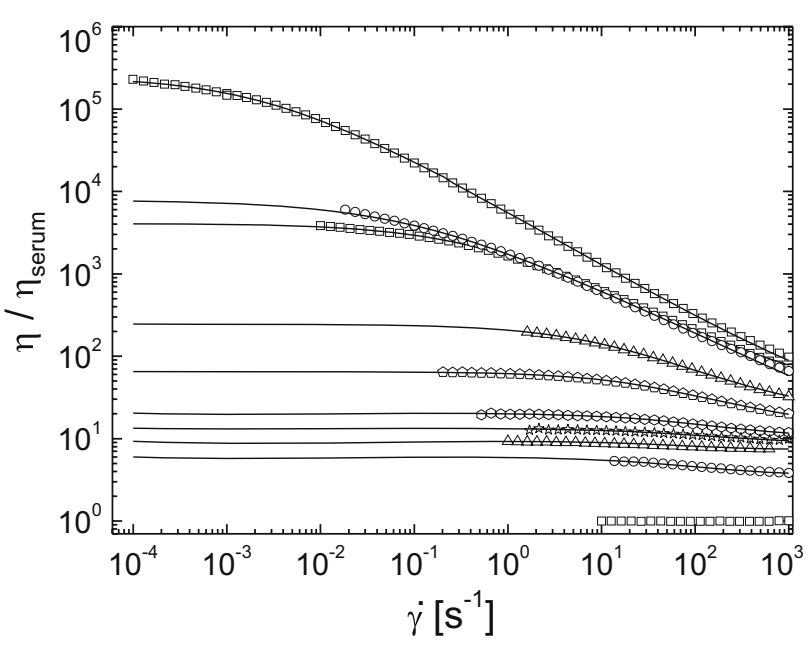

Fig. 1. Relative viscosities as a function of shear rate for casein mass percent ranging from $2.70 \%$ to $18.67 \%$ for batch II. The symbols correspond from bottom to top to casein mass percent : $2.70 \%, 6.84 \%, 10.44 \%, 11.67 \%, 12.27 \%, 13.62 \%, 15.57 \%$, $16.81 \%, 17.25 \%$ and $17.89 \%$. The solid lines are fits to the Cross-equation.

where $\eta_{\infty}$ is the high shear viscosity, $\dot{\gamma}_{c}$ is the critical shear rate at which the system starts to exhibit shear thinning. This empirical equation has been successfully used to describe the shear-thinning behavior of suspensions of hard spheres [33], red blood cells [34], and micro-gels [11,35-37]. As shown for batch II in Fig. 1, the Cross-equation perfectly fits our data up to a casein-weight percentage of $17.89 \%$, where we find that the exponent $m$ exhibits almost no dependence on concentration, $m=0.62 \pm 0.02$.

The concentration dependence of the relative zero-shear-rate viscosity is given for the two batches investigated in Fig. 2a, where the concentrations of batch I are shifted by a factor 1.19 to superimpose the data obtained with batch II. The indication of the weight fraction in Fig. 2 thus relates to the one of batch II. Since it is known that casein micelles behave like hard spheres at low to moderate concentrations [5], we show the hard sphere master curve reported by Meeker et al. [18] as a solid curve in Fig. 2a which corresponds to the relation,

$\frac{\eta_{0}}{\eta_{\text {serum }}}=\left(1-\frac{\Phi}{\Phi_{c}}\right)^{\left(-\Phi_{c}[\eta]\right)}$

with $\Phi_{c}=0.55$ and $[\eta]=2.5$. The volume fraction axis (top axis) is chosen, so that the data obtained for the milk suspensions best correspond to the behavior of the hard sphere systems in the lower concentration range. According to this rather crude mapping method, the conversion factors $q$ for batches I and II are respectively 4.4 and 3.7. The apparent divergence of the viscosity occurs at $12.25 \%$ casein content for batch I and at $14.62 \%$ casein content for batch II.

We distinguish between three distinct regimes in the concentration dependence of the viscosity. At low to moderate concentrations, regime $\mathrm{A}$, the concentration dependence of the zero-shear viscosity resembles the one of hard spheres, exhibiting an apparent divergence corresponding to an effective volume fraction of $\Phi=0.54$ assuming the $q$-values indicated above. Beyond the point of the apparent divergence, there are essential deviations from hard-sphere behavior. The zero-shear viscosity is found to remain finite. Surprisingly, between casein mass fractions of $12.5 \%$ and $13.9 \%$ for batch I and for $14.8 \%$ and $16.5 \%$ for batch II, corresponding to an effective volume fraction of 0.55 and 0.61 , within regime $B$ the viscosity changes only very little: the relative viscosity increases from 185 to 302 . Upon increasing the concentration even further, the zero-shear viscosity increases again rapidly and diverges at a concentration corresponding to an effective volume 

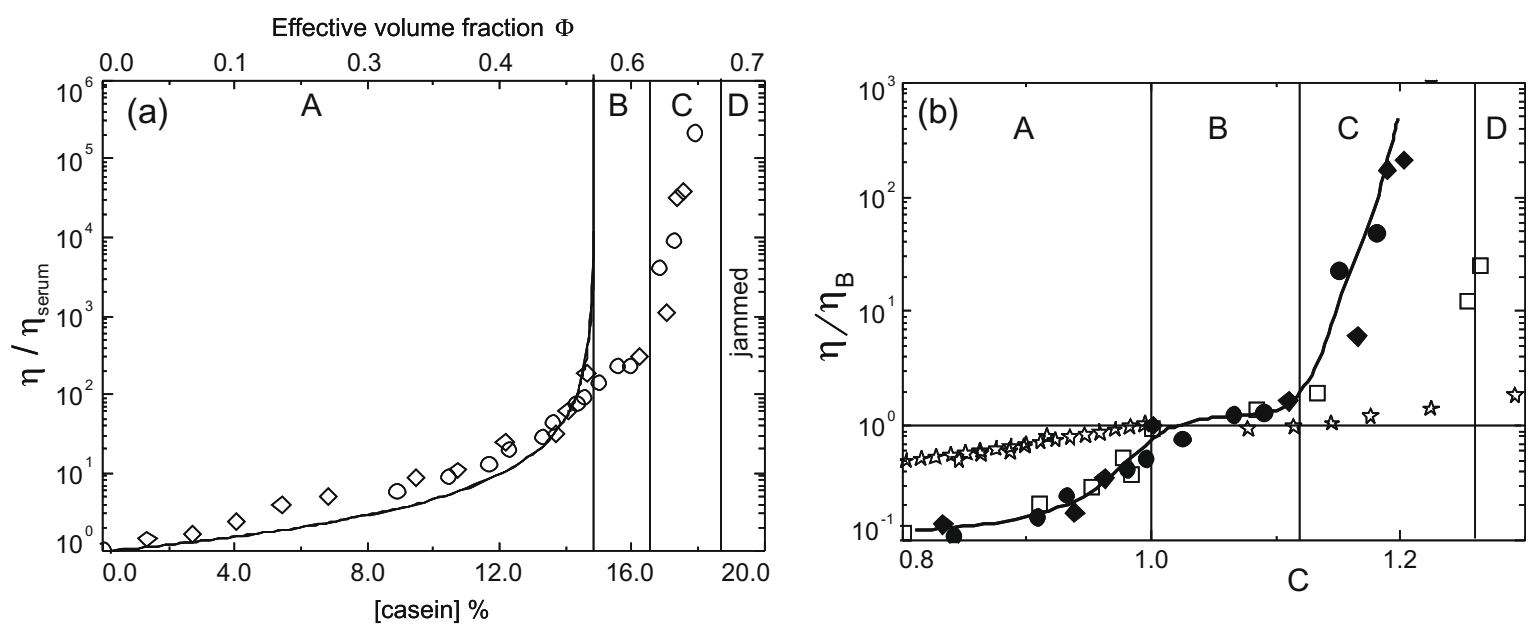

Fig. 2. (a) Relative zero-shear viscosities of the casein suspensions as a function of casein mass percent ranging from $2.70 \%$ to $18.23 \%$. The symbols $\diamond$ and $\circ$ refer to batches I and II, respectively. The mass content of batch I is multiplied by a factor 1.19 in order to display the data of both batches in a single plot. The solid line is the zero-shear viscosity for hard-sphere suspensions, as reported by Meeker [18] (corresponding to the Krieger-Dougherty model with a critical volume fraction $\Phi_{c}=0.55$ and a reduced viscosity $[\eta]=2.5$ ). The volume fraction is given on the top $x$-axis. (b) A comparison of the zero-shear viscosity of the data near regime B in (a) with those of a suspension of micro-gel particles $\square$ and star-like polymers sh, which are taken from Refs. [11,42], respectively. The concentration $C=c / c_{B}$ is the concentration scaled with the concentration $c_{B}$ where the regime $\mathrm{B}$ sets in, and the viscosity is scaled with the viscosity $\eta_{B}$ at that concentration.

fraction of 0.69 . The concentration range where the viscosity strongly increases and eventually diverges is referred to as regime $\mathrm{C}$ in Fig. 2a. Above this critical concentration, the system is structurally arrested, and is not able to flow anymore (regime D). Oscillatory rheology and DWS experiments, to be discussed in Sections 3.2 and 4 respectively, reveal independently that the system is indeed jammed in regime $\mathrm{D}$.

Deviations from hard-sphere behavior in the high concentration range have been observed for other soft colloidal systems, such as colloids with a thick layer of grafted polymers [38,40,41], micro-gel particles $[11,35,36]$, star polymers [42], sodium-caseinate solutions [43], and casein sub-micellar solutions [44]. The interpretation of this deviation is in general quite straight forward. Contrary to non-deformable hard spheres, deformable particles can still slide along each other at very high concentration by temporal changes of their shape.

The remarkable feature of the casein suspensions is that the regimes $\mathrm{A}$ and $\mathrm{C}$, where the viscosity strongly increases with concentration, are separated by a regime $\mathrm{B}$ where the viscosity changes very little with concentration. This anomaly is also found for a suspension of micro-gel particles in Refs. [11,35,36]. In order to compare the behavior of the micro-gel and our casein suspensions, the viscosities are plotted in Fig. $2 \mathrm{~b}$ as a function of the scaled concentration $C=c / c_{B}$, where $c_{B}$ is the concentration where the regime $\mathrm{B}$ sets is, that is, the concentration where the deviation from hardsphere behavior occurs. Likewise, the viscosities are scaled with the viscosity $\eta_{B}$ at the concentration $c_{B}$. As can be seen, the micro-gel suspension exhibits the same kind of behavior as the casein suspensions, in the sense that there is a regime $\mathrm{B}$, where the viscosity is essentially constant. The vertical lines in Fig. $2 b$ mark the regions for the casein suspensions. Note the extent of regime $B$ is the same for the micro-gel suspension. Also plotted in Fig. $2 \mathrm{~b}$ are data for star polymers, taken from Ref. [42]. For this extremely soft system, it is not clear whether one would be able to unambiguously define the B-regime, although there also seems to be a regime where the viscosity remains practically constant.

\subsection{Oscillatory rheological measurements}

The frequency dependence of the loss and storage modulus were measured for different concentrations in the linear viscoelas- tic regime in the frequency range of $0.1-100 \mathrm{rad} / \mathrm{s}$. In regimes $A$ and $\mathrm{B}$, the loss-modulus increases linearly with frequency while the storage modulus is too small to be measured. In regime $C$, however, the material response functions exhibit typical features of viscoelastic fluids, as shown in Fig. 3a-e. For the lowest concentration shown (16.81\%), $G^{\prime \prime}$ dominates over $G^{\prime}$ in the entire range of frequencies investigated. However, $G^{\prime}$ increases more rapidly with increasing frequencies than $G^{\prime \prime}$, which indicates that $G^{\prime}$ dominates over $G^{\prime \prime}$ at frequencies larger than the one that are experimentally accessed. Indeed, as the concentration is increased, such cross-over is observed within the frequency range investigated. The crossover frequency $\omega_{c}$ systematically decreases as the concentration increases. To evaluate $\omega_{c}$ even for the samples where we do not directly access $\omega_{c}$ experimentally, we use a scaling method. We shift all our data onto the one obtained at $17.89 \%$, in such a way that the best overlap in the low frequency range is achieved. We then use the shift factors to calculate $\omega_{c}$ and the modulus at the cross-over frequency $G_{c}=G^{\prime \prime}\left(\omega_{c}\right)=G^{\prime}\left(\omega_{c}\right)$ relative to the one obtained for $17.89 \%$, where $\omega_{c}$ and $G_{c}$ are directly accessed in the experiment. The cross-over relaxation time $\tau_{c}=2 \pi / \omega_{c}$ and the modulus at $\omega_{c}$ are given in Fig. 4 . While $G_{c}$ decreases very weakly with concentration, the relaxation time $\tau_{c}$ significantly increases upon approach of the concentration that separates the regions $C$ and $D$ in Fig. 2. Below the concentration at which the zero-shear viscosity diverges, the viscoelastic behavior is that of a liquid (see Fig. 3ad) while for larger concentrations (regime $D$ ) the material response function indicates that the system exhibits solid-like behavior; $G^{\prime}$ significantly exceeds $G^{\prime \prime}$ and exhibits an almost frequency independent behavior, as shown in Fig. 3e. This slight decrease of $G_{c}$ is attributed to the continuous decrease of the casein micelle size that takes place above $\Phi=0.55$ ( $14.86 \%$ casein weight).

\section{Diffusing wave spectroscopy}

Electric-field auto-correlation functions as determined from diffusing wave spectroscopy (DWS) measurements are shown in Fig. 5, where we have normalized the lag-time axis with $\left(L / l^{\star}\right)^{2}$ to account for the concentration dependence of $l^{\star}$. Indeed, a variation in $\left(L / l^{\star}\right)$ in a DWS experiment is equivalent to a variation of the wave vector in a DLS experiment; it leads to a change in the characteristic decay time of correlation functions, which is not 


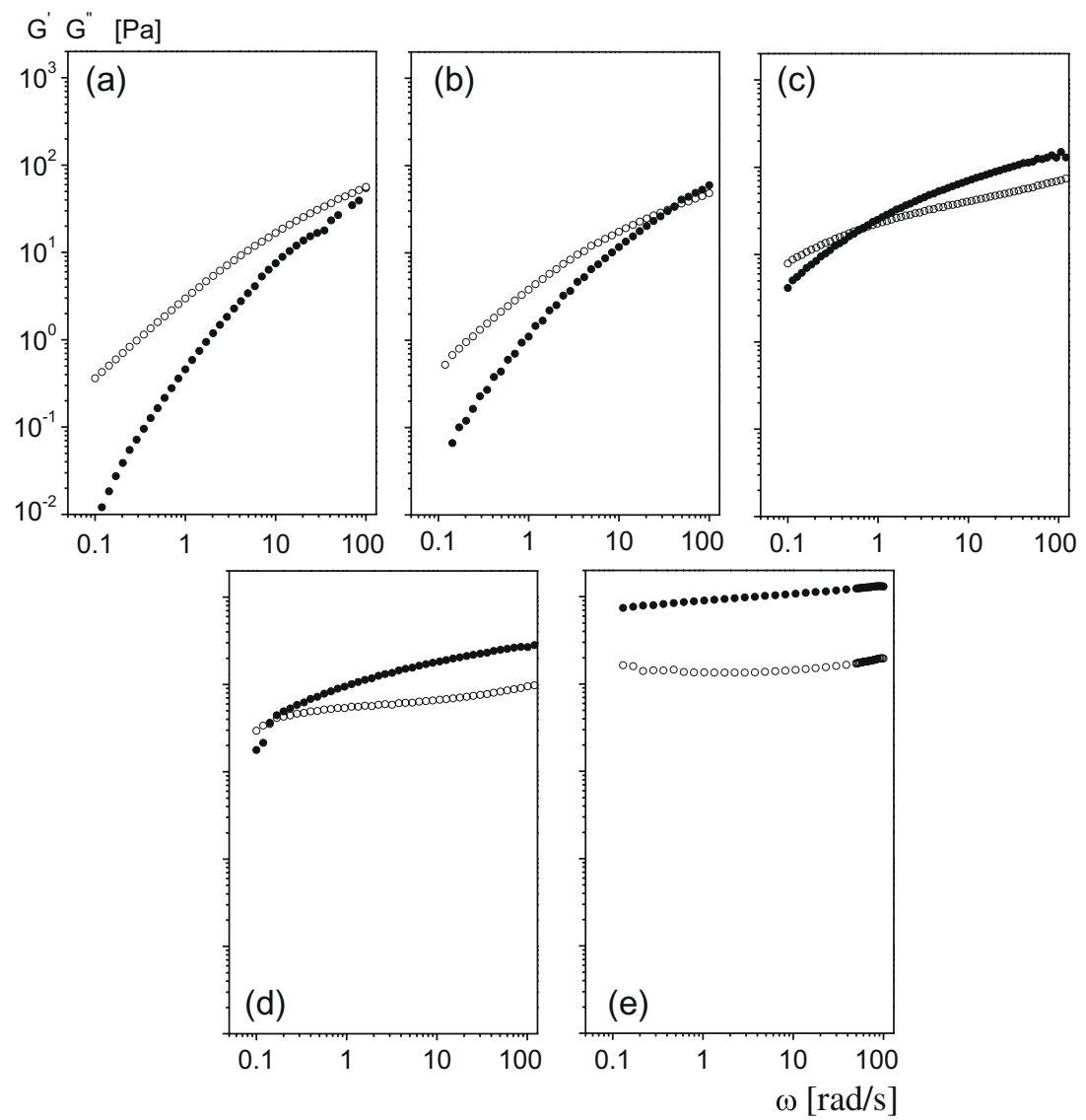

Fig. 3. (a)-(e) Storage and loss shear moduli (filled and open symbols, respectively) as a function of the angular frequency for various casein concentrations of batch II: (a) $16.81 \%, \Phi=0.62$, (b) $17.25 \%, \Phi=0.64$, (c) $17.89 \%, \Phi=0.66$, (d) $18.22 \%, \Phi=0.67$ and (e) $19.10 \%, \Phi=0.71$.

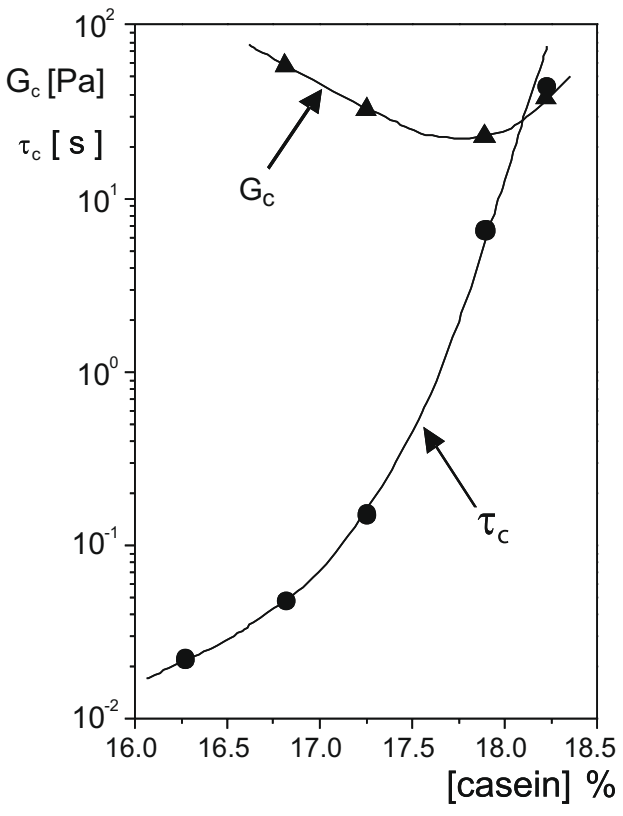

Fig. 4. The modulus $G_{c}$ at the cross-over frequency $\omega_{c}$ and the cross-over relaxation time $\tau_{c}=2 \pi / \omega_{c}$, for batch II, as a function of casein concentration.

due to a change in the dynamics of the system. To obtain comparable results it is thus necessary to account for this effect. The dependence of the correlation functions on concentration clearly shows that the dynamics of the system decreases as the concentra-

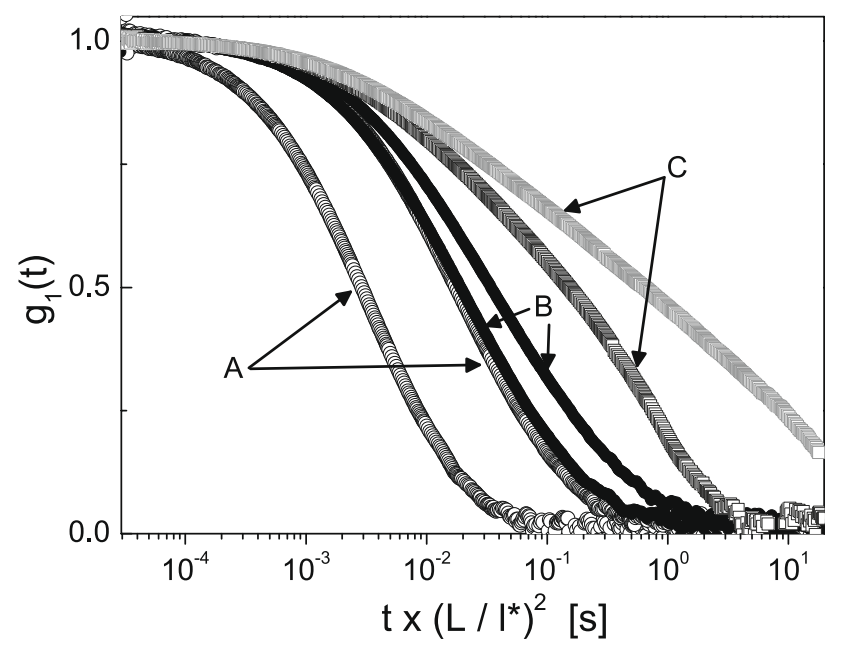

Fig. 5. Field auto-correlation functions for batch II obtained in diffusing wave spectroscopy. To account for the concentration dependence of $l^{\star}$ the lag-time axis is normalized with $\left(L / l^{\star}\right)^{2}$. From left to the right (in casein mass percent): $11.06 \%, \Phi=0.409,13.56 \%, \Phi=0.502$ (regime A, o), $14.92 \%, \Phi=0.552$, $15.57 \%, \Phi=0.576$ (regime B, •), $17.22 \%, \Phi=0.637,17.89 \%, \Phi=0.661$, (regime C, $\square)$.

tion increases. In particular, the correlation function exhibits a second decay in regime $\mathrm{C}$, which indicates the approach of the jamming transition.

In view of the ill-defined long-time behavior of the correlation functions, we determined the initial slope $-\Gamma$ of the correlation functions $g_{1}=\exp \left\{-\Gamma t\left(L / l^{\star}\right)^{2}+\mathcal{O}\left(\left(t\left(L / l^{\star}\right)^{2}\right)^{2}\right)\right\}$ to characterize 
the dynamics of our systems. Interestingly, we find that $\Gamma$ decreases continuously as the concentration is varied through the regimes A-C, as shown in Fig. 6. Particularly noteworthy is that $\Gamma$ varies quite significantly in regime $\mathrm{B}$, where the viscosity changes only slightly. Thus by contrast to the viscosity, which is an indirect measure of the structural relaxation process, $\Gamma$, which is a measure of the short-time dynamics, exhibits a monotonous dependence on concentration. This seemingly indicates that the structural relaxation process in regime $B$ is decoupled from the dynamics of the individual particles. At the threshold of the regime $B$, the casein particles start to get into contact through their kappa-casein brushes since water is no longer available in the intermicellar continuous phase [48]. When increasing further the concentration, the kappa-brushes start interpenetrating until they fully overlap. At this point, the thickness of the hairy layer is minimum and the casein micelles are close to contact. During this continuous overlapping, the volume of the particle decreases, leading to a smaller observed volume fraction, allowing the real volume fraction to remain constant in the volume-fraction range $[0.55,0.60]$. Above a volume fraction of $\Phi=0.60$ (regime C), the casein micelles are close to contact due to the collapse of their hairy kappa-brushes that became less flexible due to their compression. A further increase of concentration then leads casein micelles to deform and deswell due to osmotic compression [48]. Indeed casein micelles are soft, flexible and very loose structures composed of casein submicelles that are not densely packed, allowing the casein micelles to expel water from their inner part to the suspending medium above a threshold osmotic stress. When the caseins are no longer compressible, the system becomes dynamically arrested. Defining the jamming transition as the transition beyond which the constituents of the system touch each other, we expect the dynamics of the system to become frozen at all time scales and thus $\Gamma$ to become zero at the jamming transition. The solid line in Fig. 6 is a spline fit to the initial slope as a function of concentration, which gives a jamming concentration of $18.9 \pm 0.5 \%$. A similar extrapolation of $\omega_{c}$ to 0 is not feasible on the basis of the data obtained from Fig. 3; contrary to the DWS initial relaxation rates $\Gamma$, the rheology frequencies $\omega_{c}$ decrease strongly only in a quite small concentration range close to the jamming-transition concentration. The jamming concentration found from extrapolation of the DWS initial slopes in Fig. 6 is in between the one shown in Fig. 3d and e, where

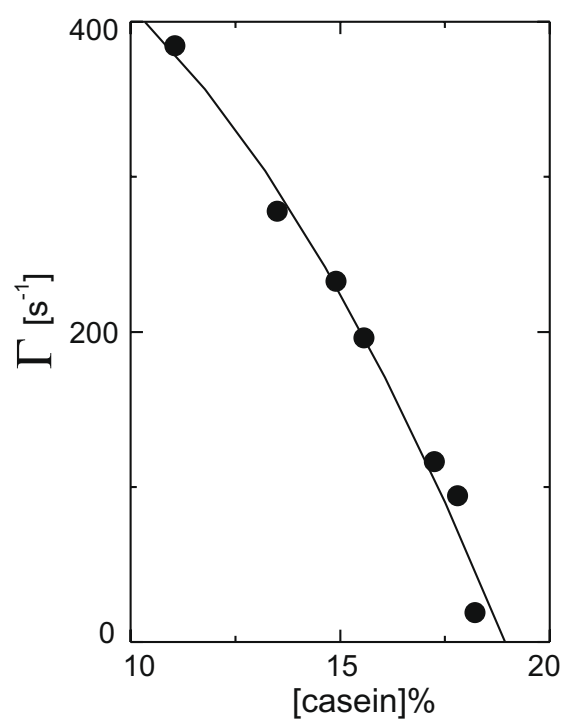

Fig. 6. The initial slope of the DWS-correlation functions as a function of the caseinweight percentage (batch II). The solid line is a sigmoidal fit, from which the jamming-transition concentration is found to be equal to [casein] $=(18.9 \pm 0.5) \%$. the oscillatory rheology data reveal a transition from liquid-like to solid-like behavior. It also agrees with the estimated concentration in Fig. 2a that separates regime C from regime D. We can therefore conclude that within regime D in Fig. 2a the system is jammed.

Since in the regime B the true volume fraction remains essentially unchanged in the range of volume fractions $[0.55,0.60]$, all the volume fractions in the regime $C$ should be shifted by a constant of 0.05 to a lower value, so that the true volume fraction at which the jamming occurs is $0.64(=0.69-0.05)$. This indeed corresponds to the volume fraction at which a liquid of hard spheres undergoes a transition to the glassy state.

\section{Conclusions}

Concentrated industrial casein suspensions are shown to exhibit unexpected viscous behavior at very high casein content. The hard sphere like behavior at moderate casein content allows to express casein concentrations in terms of an effective volume fraction. Hard sphere like behavior is found up to volume fractions of 0.54 (this regime is referred to as regime A). An unexpected weak concentration dependence of the viscosity is found in the volumefraction range $0.55-0.60$ (regime $B$ ). At volume fractions above 0.60 , there is a much steeper increase of the viscosity with increasing concentration, and the zero-shear viscosity eventually diverges at a limiting volume fraction of 0.69 (regime C). Oscillatory rheology measurements of the storage and loss moduli show that the caseins behave more and more solid-like when the concentration approaches this limiting concentration. In addition, diffusive wave spectroscopy (DWS) shows that short-time diffusion becomes very slow close to this limiting concentration. The initial slope of DWScorrelation functions vanishes, to within experimental error, at the same concentration of $\Phi=0.69$ where the zero-shear viscosity diverges. This indicates that above this concentration (regime $D$ ) the system is in a structurally arrested state.

The anomalous behavior within regime $\mathrm{B}$, where the viscosity remains essentially constant, independent of concentration, is also observed for suspensions of micro-gel particles $[11,35,36]$. On the basis of an independent investigation of the deswelling behavior of casein micelles [48], one possible reason for the constant viscosity in regime $B$ for the casein micellar systems is that the volume of the micelles decreases due to osmotic compression. On the other hand, the initial slope of DWS-correlation functions shows, in contrast to the low-shear viscosity, a quite monotonic behavior as a function of concentration through the regimes A-C. This indicates that there is no size change or a change in shape fluctuations of individual particles that could cause the anomalous weak concentration dependence of the low shear viscosity in regime $B$. The anomalous behavior in regime $B$ could thus also be due to the response of large scale structures, extending over distances much larger than the size of single caseins. To resolve this issue, for both the casein micelles and micro-gel particle suspensions, further investigations on both the particle size and large-scale microstructural arrangement under weak flow conditions should be done, for example by means of neutron scattering and electron microscopy.

\section{Acknowledgment}

We gratefully acknowledge financial support from the Swiss National Science Foundation.

\section{References}

[1] R. Mezzenga, P. Schurtenberger, A. Burbidge, M. Michel, Nature Mater. 4 (2005) 729. 
[2] P. Schurtenberger, A. Stradner, S. Romer, C. Urban, F. Scheffold, Chimia 55 (2001) 155.

[3] C. Holt, Adv. Protein Chem. 43 (1992) 63.

[4] C. Holt, C.G. De Kruif, R. Tuinier, P.A. Timmins, Colloids Surf. A 213 (2003) 275.

[5] C.G. de Kruif, J. Dairy Sci. 81 (1998) 3019

[6] C.G. de Kruif, Langmuir 8 (1992) 2932.

[7] M. Alexander, L.F. Rojas-Ochoa, M. Leser, P. Schurtenberger, J. Colloid Interface Sci. 253 (2002) 35

[8] P. Walstra, J. Dairy Sci. 73 (1990) 1965.

[9] P.X. Qi, J. Dairy Sci. Technol. 87 (2007) 363.

[10] C.G. De Kruif, C. Holt, in: P.F. Fox, P.L.H. McSweeney (Eds.), Advanced Dairy Chemistry, Proteins, third ed., vol. 1, Kluwer Academic/Plenum Publishers, 2003.

[11] H. Senff, W. Richtering, J. Chem. Phys. 111 (1999) 1705.

[12] P. Mills, J.M. Rubi, D. Quemada, Rheology 2 (1980) 127.

[17] P. Walstra, J. Dairy Res. 2 (1979) 317.

[18] S.P. Meeker, W.C.K. Poon, P.N. Pusey, Phys. Rev. E 55 (1997) 5718

[19] S.E. Phan, W.B. Russel, Z.D. Cheng, J.X. Zhu, P.M. Chaikin, J.H. Dunsmuir, R.H. Ottewill, Phys. Rev. E 54 (1996) 6633

[20] G.J. Brownsey, T.R. Noel, R. Parker, S.G. Ring, Biophys. J. 85 (2003) 3943.

[21] G. Maret, Curr. Opin. Colloid Interface Sci. 2 (1997) 251.

[22] J.X. Zhu, D.J. Pine, D.A. Weitz, Phys. Rev. A 44 (1991) 3948

[23] A.J. Vasbinder, A.C. Alting, K.G. De Kruif, Colloid Surf., B 31 (2003) 115.

[24] D.G. Dalgleish, M. Alexander, M. Corredig, Food Hydrocolloids 18 (2004) 747.

[25] M. Alexander, D.G. Dagleish, Langmuir 21 (2005) 11380.
[26] Y. Hemar, H. Singh, D.S. Horne, Curr. Appl. Phys. 4 (2004) 362.

[27] M. Alexander, M. Corredig, D.G. Dalgleish, Food Hydrocolloids 20 (2006) 325.

[28] For more information on this set up, see <http://www.lsinstruments.ch/>

[29] S. Romer, F. Scheffold, P. Schurtenberger, Phys. Rev. Lett. 23 (2000) 4980.

[30] F. Scheffold, S.E. Skipetrov, S. Romer, P. Schurtenberger, Phys. Rev. E 63 (2001) 061404

[31] L.F. Rojas-Ochoa, S. Romer, F. Scheffold, P. Schurtenberger, Phys. Rev. E 65 (2002) 051403.

[32] M.M. Cross, J. Colloid Sci. 20 (1965) 417.

[33] D.E. Quemada, in: B. Mena (Ed.), Advances in Rheology: Fluids, vol. 2, Univ. Nac. Aut. de Mexico, Mexico City, 1984

[34] P. Mills, J.M. Rubi, D. Quemada, Rheology (Fluids, Plenum Press) 2 (1980) 127.

[35] H. Senff, W. Richtering, Langmuir 15 (1999) 102.

[36] H. Senff, W. Richtering, Colloid Polym. Sci. 278 (2000) 830

[37] D.A.R. Jones, B. Leary, D.V. Boger, J. Colloid Interface Sci. 147 (1991) 479.

[38] P. Auroy, L. Auvray, L. Lger, J. Phys.: Condens. Matter 2 (1990) 317.

[40] P.A. Nommensen, M.H.G. Duits, J.S. Lopulissa, D. Van den Ende, J. Mellema, Prog. Colloid Polym. Sci. 110 (1998) 144.

[41] J. Mewis, AIChE J. 35 (1989) 415.

[42] J. Roovers, Macromolecules 27 (1994) 5359.

[43] D. Farrer, A. Lips, Int. Dairy J. 9 (1999) 281.

[44] M. Panouille, L. Benyahia, D. Durand, T. Nicolai, J. Colloid Interface Sci. 287 (2005) 468.

[48] A. Bouchoux, P.E. Cayemitte, J. Jardin, G. Gesan-Guiziou, B. Cabane, Biophys. J. 96 (2009) 693. 